\title{
3D GIS FOR FLOOD MODELLING IN RIVER VALLEYS
}

\author{
P. Tymkow*, M. Karpina, A. Borkowski \\ Institute of Geodesy and Geoinformatics, Wroclaw University of Environmental and Life Sciences, Poland, - (przemyslaw.tymkow, \\ mateusz.karpina, andrzej.borkowski)@igig.up.wroc.pl
}

\author{
Commission VIII, WG VIII/1
}

KEY WORDS: 3D GIS, flood modelling, CityGML, laser scanning, 3D geometry modelling

\begin{abstract}
:
The objective of this study is implementation of system architecture for collecting and analysing data as well as visualizing results for hydrodynamic modelling of flood flows in river valleys using remote sensing methods, tree-dimensional geometry of spatial objects and GPU multithread processing. The proposed solution includes: spatial data acquisition segment, data processing and transformation, mathematical modelling of flow phenomena and results visualization. Data acquisition segment was based on aerial laser scanning supplemented by images in visible range. Vector data creation was based on automatic and semiautomatic algorithms of DTM and 3D spatial features modelling. Algorithms for buildings and vegetation geometry modelling were proposed or adopted from literature. The implementation of the framework was designed as modular software using open specifications and partially reusing open source projects. The database structure for gathering and sharing vector data, including flood modelling results, was created using PostgreSQL. For the internal structure of feature classes of spatial objects in a database, the CityGML standard was used. For the hydrodynamic modelling the solutions of Navier-Stokes equations in two-dimensional version was implemented. Visualization of geospatial data and flow model results was transferred to the client side application. This gave the independence from server hardware platform. A real-world case in Poland, which is a part of Widawa River valley near Wroclaw city, was selected to demonstrate the applicability of proposed system.
\end{abstract}

\section{INTRODUCTION}

\subsection{Motivation}

In recent years a number of implementations in the field of spatial data acquisition have been carried out, usually in the form of point sets or close-range photogrammetric images. They are used for 3D spatial data infrastructure development. With the algorithms of automatic and semiautomatic spatial objects modelling and development in the field of data acquisition techniques, it became possible to create object-oriented GIS 3D and their efficient update. To ensure interoperability of these systems, certain standards are being set for 3D data such as CityGML. Using topological data model supplemented by attribute information, the application of such systems in modelling natural phenomena is possible. Especially significant potential can be seen in the case of flood simulation. However, this requires improvement in data integration, its processing and the mathematical simulation models.

The importance of the numerical models of such phenomena as flood inundation and water wave propagation for the scientific research, administrative decisions, designing or crisis management is widely recognised. Particular importance, given the range and scope of environmental damage, lays in the systems used to support the prediction and the effects of flooding. This especially relates to strongly-urbanised areas. Modern measurement techniques used in geodesy and in remote sensing allow for the creation of three-dimensional models of space that are more effective in reflecting the reality than still more frequently used spatial models which have the form of two-dimensional maps and spatial information systems. This particularly applies to objects such as buildings and structures or vegetation. Creating a virtual reality based on 3D geometry helps to facilitate the perception of such models and at the same time can provide spatial frameworks for the analyses of more detailed presentation. Software packages used in hydrodynamic modelling such as e.g. MIKE may use 3D geometric data to a small degree. They are based on the $2.5 \mathrm{D}$ or descriptive data. However, the potential of $3 \mathrm{D}$ data is ample. This applies not only to visualisation, but also to the boundary conditions of simulations themselves. With the increase of computing power of computers, it is possible to use a detailed geometry of spatial objects instead of simplified models or descriptive parameters of e.g. roughness, to a greater degree. Works on visualisation systems and on sharing the results of the simulations with GIS environment have been carried out for a long time (e.g.: Clark, 1998; Ng et al., 2009, 2007; Vivoni and Richards, 2005; Yu et al., 2012). In order to integrate a hydrodynamic model with the GIS system, it is necessary to match the method of geospatial data representation to the way in which the simulation is implemented, as well as to standardise the data and optimise the calculations. Works on optimising the hydrodynamic calculations on the graphics processing units constitute the subject of numerous studies (e.g. Horváth et al., 2016).

The article briefly presents the assumptions and the method of the implementation of a pre-prototypical version of the proprietary GIS 3D system, the aim of which was to adapt the requirements for 3D spatial data to their use in hydrodynamic modelling and for presentation of the results of this modelling in such systems. Presented system was realized with the implementation for the graphical processor units (GPU).

\footnotetext{
* Corresponding author
} 


\subsection{Geospatial data representation in various types of hydrodynamic modelling}

In hydrodynamic modelling of flood flows, one of the most important part is the geospatial data and hence the data about the shape of the surface of the riverbed and valley as well as the information about the flow resistance. Depending on the method of hydrodynamic modelling and the degree of simplicity of such a model, the land and the real spatial objects are presented at various levels of generalisation. Basically, the following hydrodynamic models may be distinguished: one-, two- and three-dimensional. One-dimensional systems, and hence the ones based on the assumption that the speed component compatible with the direction of the axis of the river constitutes the dominant one, use the description of the geometry of the flow area in the form of the cross-sections which are perpendicular to the direction of the flow. In the case of such an approach, the impact of land-use objects, such as buildings or vegetation, on the wave distribution is usually expressed through alternative resistance factors. 2D systems are used in the simulation of the flows in the riverbeds, where the vertical component of the speed and acceleration vectors are much smaller than the components in the horizontal plane. The characteristics of motion parameters are therefore determined by the longitudinal coordinate. The example of such phenomena are so-called flash floods. The geometry of the valleys and the riverbed in these models is represented with the use of the DTM (2.5D geometry) whereas the roughness coefficients, which amongst others include resistance associated with vegetation, are presented in a spatial manner by referring them to the individual meshes of DTM grid.

3D models allow for a complete description of the flow, taking into account all the components of the velocity vector. They were widely used to simulate the flow of the turbulent heat or mass fluxes. One of the numerical methods for the water mass flow in the assumed boundary conditions is simulation in the form of fluid particles, whereas the Nivera-Stokes equation constitutes the most commonly used equation for describing motion. In order to solve the equations of the liquid masses motion in the form of fluid particles, both the land and the elements of the land use and cover may be modelled with a high level of detail. A fully three-dimensional geometry of their description is admissible. GIS software (Merwade et al., 2008) is most commonly used in the preparation of geospatial data.

\subsection{Standardisation of data}

Spatial data in 3D GIS systems may be presented at various levels of generalisation and contain various semantic information. Their structure may be topologically accurate or not. They may also contain the attribute component. In order to build a system that would enable us to perform analyses based on such data, the data must be subject to standardisation. CityGML (OGC, 2012, Kolbe et al., 2005) was selected as a standard for this project. This standard defines the classes and their relations for the most relevant objects in urban areas with respect to their geometrical, topological, semantic and appearance properties. It introduces generalisation hierarchies, aggregations, associations between objects, and spatial properties. CityGML standard is based on GML language. Therefore, the following geometrical elements are used in modelling the objects: Point, Curve, Surface and Solid. These elements are adapted according to specific conditions described in the standard. The basic concepts included in CityGML are levels of detailed presentation of objects (Level of Detail), which represent the same space at various levels of generalisation. On the basis of the guidelines included in the standard, and partly on the basis on its own amplification for objects not described therein such as levels of detail for vegetation, the geometry modelling algorithms were implemented. Thanks to the unitary record of the data structure, firstly, it is possible to integrate various solutions and technologies in the project and secondly, the project gains the interoperability characteristics.

\section{THE CONCEPT OF 3D GIS SYSTEM FOR HYDRODYNAMIC ANALYSES}

\subsection{General assumptions}

Basically, the whole system consists of three main components: a software part which constitutes a group of both proprietary and external software for managing data, performing analyses as well as for data modelling and standardisation. The second component is a relational database whereas the third includes the measuring and external data import segments (Figure 1).

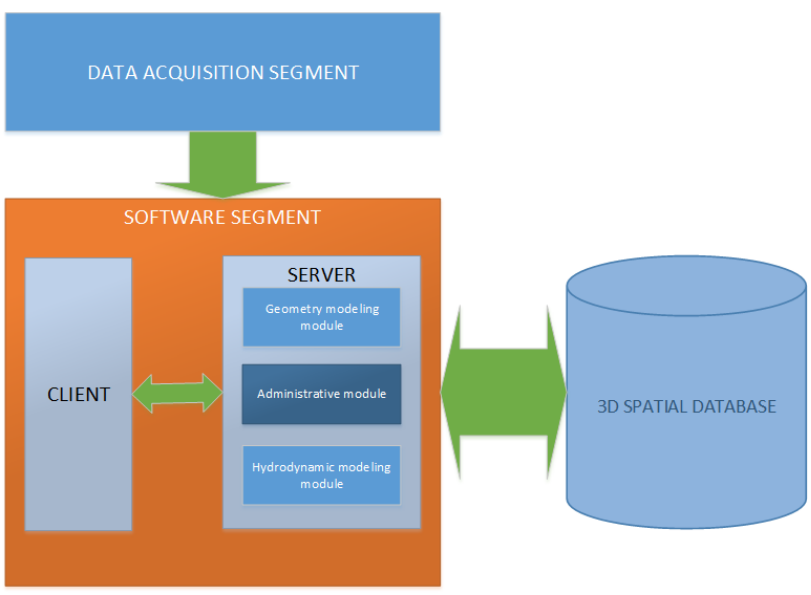

Figure 1. Conceptual diagram of the system

A client-server architecture type with a modular structure constitutes the basic premise of the software section design. Windows system constitutes its working environment. The client part includes a desktop application with the function to visualise the spatial data chosen arbitrarily from the database (in the form of thematic layers) as well as the function to define the parameters of the hydrodynamic simulation and to visualise the very simulation. On the server side, there is a group of modules dedicated to different tasks. Each of the modules is developed independently by using different technologies. The element which links each module is the administrative module, which provides communication between the client part and the hydrodynamic modelling module. Data modelling modules comprise a set of tools and external software, not integrated with one graphical interface for the whole system at the present stage of the work. However, thanks to the standardisation of the output data, it is possible to exchange and supply the spatial database directly.

Part of the data acquisition is a set of measurement techniques that provide data in the form of a cloud of points. As the primary source of data, it was decided to use the data from airborne and terrestrial laser scanning. In addition, some algorithms of LIDAR geometry modelling data are supported by other data such as, for example: 2D information about outlines 
of buildings which stem from the reference database of topographic objects or orthophotomaps.

\subsection{Implementation}

2.2.1 The server part: Due to the use of libraries that support multithreaded calculations on graphics processing units (GPU), part of the hardware for the server modules is limited to Nvidia technology-based systems. The implementation of this part was made for nVidia Tesla M4090. It is equipped with 2048 GPU.

2.2.2 Hydrodynamic modelling: The first attempts to implement the hydrodynamic model in the system were based on the Nivera-Stokes equation for the shallow water:

$$
\left.\begin{array}{c}
\frac{\partial H}{\partial t}+\operatorname{div}(H w)=0 \\
\frac{\partial H w}{\partial t}+\operatorname{div}(H w w)+\frac{g}{2} \nabla H^{2}=H g \nabla d
\end{array}\right\}
$$

where: $\mathrm{H}$ - water depth, w - velocity vector, g - gravitational constant, $\mathrm{d}$ - water depth measured from the surface of the water table.

The finite differences method was used to solve the equation. The height of the mirror of water, and speed in the nodes of the numerical model of land cover grid, created on the basis of DTM and solid models of buildings in the database in the LOD1 standard were calculated. C++ language and OpenCL libraries were used. The next stage of the work will be the integration of 3D hydrodynamic model based on the fluid particles, which allows for the use of larger amounts of spatial data.

2.2.3 Desktop client application: The graphical user interface of the client application is built on the basis of a system of docked elements of AvalonDock library. The main menu has mainly a ribbon style. It is divided into two tabs: document and simulation. The first tab contains operations for the current document and the program window. It is possible to add layers, change the camera view and the settings in your 3D scene rendering. The second tab refers to simulation. An exemplary view of an open document, together with a running simulation for the part of Widawa River Valley (Southwest Poland) movement is presented in Figure 2.

2.2.4 Database: In order to store the data, the relational database "3dcitydb" was chosen in the implementation of the PostGIS. It constitutes the transition of CityGML data structures into the tables in the relational data model.

2.2.5 3D geometry modelling module: Numerical terrain models, which describe in detail the position of the edge line of the surface of the land in the area of the river valley, for instance river embankments, are necessary for hydrodynamic modelling. DTM are built mostly on the basis of the data from airborne laser scanning. On the basis of an irregular set of laser scanning points, 3D edge lines can also be modelled. Edge lines on river embankments are characterised by their blurriness. In addition, they are often hidden in the underbrush or in high vegetation.

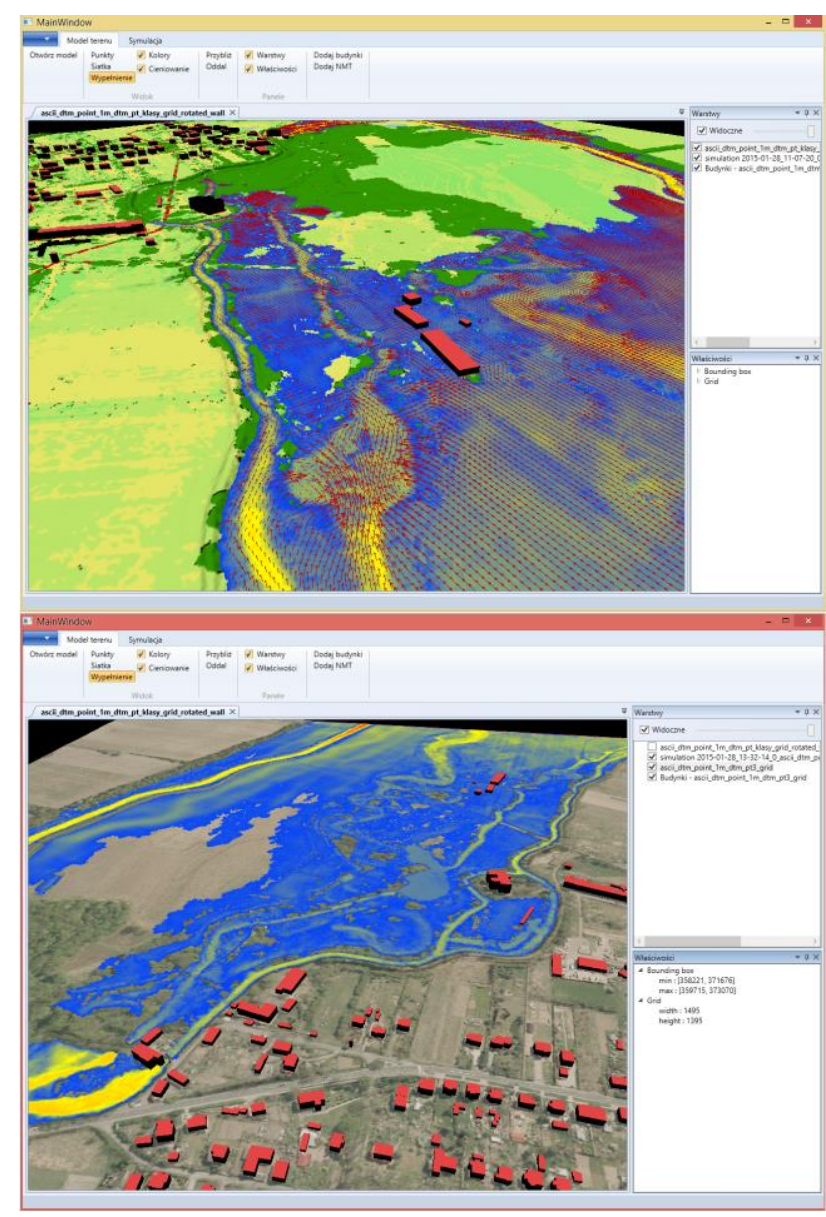

Figure 2. Desktop client interface

The project uses the algorithm of modelling the edge line as the intersection line of two surfaces, in which laser scanning point data located on both sides of the modelled edge are approximated (Borkowski and Keller, 2005). Thin plate splines were used as a functional model of the surface. The position of the line in the xy plane is determined in the evaluated algorithm with the use of active contour model (snakes), where the external energy in the snakes model is defined as proportional to the distance between both surfaces in the current position of the active contour. The third coordinate of the edge line is obtained by inserting the $x y$ coordinates of the Snake model into the functional model of approximating surface. The algorithm requires the classification of laser scanning points into the areas lying on both sides of the modelling edge. The controlled classification based on multilayer feed-forward neural networks was used to achieve this. Information about the shape and orientation of the surface, which is identified in relation to the river, was included in the process. Models of buildings were modelled with LoD1 and LoD2 accuracy. LoD1 is used to create the grid (DSM), where the simulation of a twodimensional hydrodynamic model takes place. LoD2 models are used to visualise the simulation and can be applied directly to hydrodynamic 3D simulation. At LoD1 level, the buildings are simple solids, for creation of which the information about the height and the shape of the outline on the ground surface is necessary. The calculation of the roof height was implemented in the form of the processing scheme (Model Builder) in the ArcGIS environment on the basis of airborne laser scanning data, DTM data and 2D information about the outlines of the buildings which stem from referential database of topographical 
objects (BDOT10k). LoD2 models were created with the use of automatic method based on the algorithm presented in the work of Jarząbek-Rychard (Jarząbek-Rychard, 2012). Airborne laser scanning points are used as data, with no additional information. Building model is retained due to the topological relationships between the characteristic points which identify particular edge lines of roofs and walls. All 3D models of buildings have undergone standardisation according to CityGML. CityEditor tool was used for Skechup.

The geometry of the vegetation (trees and larger shrubs) was modelled by using the algorithm based on convex-hull method (Tymkow and Borkowski, 2010). Modelling method allows for the creation of plant geometry as a macrostructural model that is a compact solid, the shape of which is determined by the outline of external points and as a microstructural model, meaning the reconstructions of the trunk and larger branches geometry. 3D models of vegetation are not used in two-dimensional modelling, due to the fact that such modelling is based on the description of the geometry of the valley in the form of $2.5 \mathrm{D}$ grid. They can be used while implementing the 3D modelling module into the system. The first attempts to use 3D data from the spatial database system in modelling with the use of fluid particles were carried out with the use of Fluidix software (Figure 3).

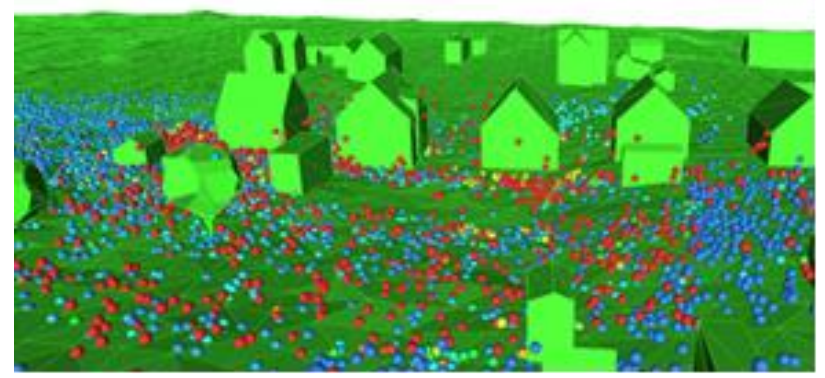

Figure 3. The use of 3D spatial data (DTM, models of buildings and trees) in the hydrodynamic 3D simulation of fluid particles type.

\section{CONCLUSIONS}

Building spatial framework for simulating flood phenomena requires solving a number of minor problems such as optimization algorithms to create and generalization of spatial information based on measured data, standardization of such information or fitting scale and resolution to the model of flow phenomena. In this study the 3D spatial information system is developed and implemented that meet these requirements.

The developed spatial information system is open, fully independent solution dedicated for hydrodynamic modelling of floods in river valley. In contrary to existing system the proposed system deploy 3D GIS objects into system framework. Thanks to multilevel spatial data generalization and modular architecture the system allows to use different hydrodynamic models.

\section{ACKNOWLEDGEMENTS}

The outcome has been achieved with the financial support of National Science Centre, Poland granted on the basis of the decision No. DEC2011/01/D/ST10/07671.

\section{REFERENCES}

Borkowski, A., Keller, W., 2005. Global and local methods for tracking the intersection curve between two surfaces. Journal of Geodesy 79, pp. 1-10.

Clark, M.J., 1998. Puting water in its place: a perspective on GIS in hydrology and water management. Hydrological Processes 12, pp. 823-834.

Horváth, Z., Perdigão, R.A., Waser, J., Cornel, D., Konev, A., Blöschl, G., 2016. Kepler shuffle for real-world flood simulations on GPUs. International Journal of High Performance Computing Applications, pp. 1-17.

Jarząbek-Rychard，2012. Automatic reconstruction of 3d building skeleton models based on airborne laser scanning data. (in polish) Archiwum Fotogrametrii, Kartografii i Teledetekcji 24, pp. 99-108.

Kolbe, T.H., Gröger, G., Plümer, L., 2005. CityGML: Interoperable access to 3D city models, in: Geo-Information for Disaster Management. pp. 883-899.

Merwade, V., Cook, A., Coonrod, J., 2008. GIS techniques for creating river terrain models for hydrodynamic modeling and flood inundation mapping. Environmental Modelling and Software 23, pp. 1300-1311.

Ng, S.M.Y., Wai, O.W.H., Li, Y.-S., Li, Z.-L., Jiang, Y., 2009. Integration of a GIS and a complex three-dimensional hydrodynamic, sediment and heavy metal transport numerical model. Adv. Eng. Softw. 40, 391-401.

Ng, S.M.Y., Wai, O.W.H., Li, Y.S., Xu, Z.H., Chen, H.L., Li, Z.L., 2007. Development of a GIS for managing dynamic, 3D coastal information of Pearl River Estuary. Journal of Hydroinformatics 9, pp. 215-232.

OGC, 2012. OGC City Geography Markup Language (CityGML) Encoding Standard v. 2.0. Open Geospatial Consortium 12-019.

Tymkow, P., Borkowski, A., 2010. Vegetation modelling based on TLS data for roughness coefficient estimation in river valley. International Archives of the Photogrammetry, Remote Sensing and Spatial Information Sciences , pp. 309-313.

Vivoni, E.R., Richards, K.T., 2005. Integrated use of GIS-based field sampling and modeling for hydrologic and water quality studies. J. Hydroinform. 7, pp. 235-250.

Yu, J.J., Qin, X.S., Larsen, L.C., Larsen, O., Jayasooriya, A., Shen, X.L., 2012. A GIS-based management and publication framework for data handling of numerical model results. $A d v$. Eng. Softw. 45, pp. 360-369. 